Pacific Journal of Mathematics

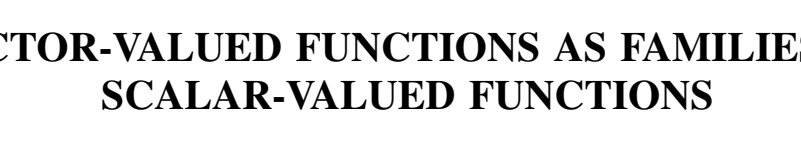




\title{
VECTOR-VALUED FUNCTIONS AS FAMILIES OF SCALAR-VALUED FUNCTIONS
}

\author{
ROBERT F. Geitz AND J. J. UhL, JR.
}

One time-honored way of studying the properties of a vector measure $F$ with values in a Banach space $X$, with dual $X^{*}$ is to examine properties of the family of scalar measures $\left\{\left\langle x^{*}, F\right\rangle: x^{*} \in X^{*},\left\|x^{*}\right\| \leqq 1\right\}$. The purpose of this paper is to undertake a similar study for vector-valued functions. The first theorem proved in this vein was the classical Pettis measurability theorem which states that if $X$ is a separable Banach space and $f$ is an $X$-valued function such that $\left\langle x^{*}, f\right\rangle$ is measurable for each $x^{*}$ in $X^{*}$, then $f$ is a measurable function. What we propose to do is to take a bounded function $f$ with values in $X$, form the associated family $\mathscr{F}=\left\{\left\langle x^{*}, f\right\rangle: x^{*} \in X^{*},\left\|x^{*}\right\| \leqq 1\right\}$ and study how measurability and integrability properties of $f$ are reflected by topological properties of $\mathscr{T}$ in the spaces $L_{\infty}$ and $B(\Sigma)$.

Throughout this paper $(\Omega, \Sigma, \mu)$ is a finite measure space and $X$ is a Banach space with dual $X^{*}$. A function $f: \Omega \rightarrow X$ is $(\mu-)$ measurable if it is the a.e. limit of a sequence of simple functions. Standard arguments show that if $\mu$ is a Radon measure, then $\mu$ measurablity is equivalent to Lusin $\mu$-measurability, which means that for every compact set $K \subset \Omega$ and for every number $\delta>0$ there is a compact set $K^{\prime} \subset K$ such that $\mu\left(K \backslash K^{\prime}\right)<\delta$ and $f$ restricted to $K^{\prime}$ is continuous. The function $f$ is weakly ( $\mu$-) measurable if $\left\langle x^{*}, f\right\rangle$ is measurable for all $x^{*}$ in $X^{*}$. If $X$ is the dual of a space $Y$, then $f$ is weak*-( $\mu-)$ measurable if $\langle f, y\rangle$ is measurable for all $y$ in $Y$. A function $f: \Omega \rightarrow X$ is weakly equivalent to a function $g: \Omega \rightarrow X$ if $\left\langle x^{*}, f\right\rangle=\left\langle x^{*}, g\right\rangle$ a.e., for every $x^{*}$ in $X^{*}$. If $X$ is the dual of space $\mathscr{Y}$, then $f$ and $g$ are weak*-equivalent if $\langle f, y\rangle=\langle g, y\rangle$ a.e., for all $y$ in $\mathscr{Y}$.

A weakly measurable function $f: \Omega \rightarrow X$ is Pettis integrable if for each $E$ in $\Sigma$ there is an element $P-\int_{E} f d \mu$ in $X$ such that

$$
x^{*}\left(P=\int_{E} f d \mu\right)=\int_{E} x^{*} f d \mu .
$$

1. The family $\left\{\left\langle x^{*}, f\right\rangle:\left\|x^{*}\right\| \leqq 1\right\}$ as a subset of $L_{\infty}(\mu)$. In this section we shall study a bounded function $f: \Omega \rightarrow X$ in terms of topological properties of the associated family $\left\{\left\langle x^{*}, f\right\rangle:\left\|x^{*}\right\| \leqq 1\right\}$ as a subset of the space $L_{\infty}(\mu)$. 
For the first lemma recall that $\left(L_{\infty}(\mu)\right)^{*}$ consists of all finitely additive bounded signed measures on $\Sigma$ that vanish on $\mu$-null sets.

Lemma 1. If $f: \Omega \rightarrow X$ is bounded, weakly measurable and Pettis integrable with respect to all $\lambda$ in $\left(L_{\infty}(\mu)\right)^{*}$, then $\left\{\left\langle x^{*}, f\right\rangle:\left\|x^{*}\right\| \leqq 1\right\}$ is a relatively weakly compact subset of $L_{\infty}(\mu)$.

Proof. Let $\left(\left\langle x_{\alpha}^{*}, f\right\rangle\right)$ be a net in $\left\{\left\langle x^{*}, f\right\rangle:\left\|x^{*}\right\| \leqq 1\right\}$ and select a weak*-convergent subset $\left(x_{\beta}^{*}\right)$ of $\left(x_{\alpha}^{*}\right)$. Let $x_{0}^{*}$ be the weak-limit of $\left(x_{\beta}^{*}\right)$ and observe that for $\lambda$ in $L_{\infty}(\mu)^{*}$ one has by Pettis integrability

$$
\begin{aligned}
\lim _{\beta} \int_{\Omega}\left\langle x_{\beta}^{*}, f\right\rangle d \lambda & =\lim _{\beta}\left\langle x_{\beta}^{*}, P-\int_{\Omega} f d \lambda\right\rangle \\
& =\left\langle x_{\nu}^{*}, P-\int_{\Omega} f d \lambda\right\rangle=\int_{\Omega}\left\langle x_{\nu}^{*}, f\right\rangle d \lambda .
\end{aligned}
$$

Thus $\left(x^{*}, f\right)$ has a weakly convergent subnet and the proof is complete.

The first part of the following theorem is well-known (see [1, p. 88]). Its proof is reproduced here mainly to obtain an easy proof of the second part.

THEOREM 2. If $f: \Omega \rightarrow X$ is bounded and weakly measurable and if the set $\left\{\left\langle x^{*}, f\right\rangle:\left\|x^{*}\right\| \leqq 1\right\}$ is relatively weakly compact in $L_{\infty}(\mu)$, then there exists a measurable function $g: \Omega \rightarrow X^{* *}$ such that $f$ is weak*-equivalent to $g$.

If, in addition, the function $f$ is Pettis integrable with respect to $\mu$, then there is a measurable function $g: \Omega \rightarrow X$ such that $f$ is weakly equivalent to $g$.

Proof. Define an operator $T: X^{*} \rightarrow L_{\infty}(\mu)$ by $T x^{*}=\left\langle x^{*}, f\right\rangle$. By hypothesis, the operator $T$ is weakly compact and by Gantmacher's theorem so is its adjoint $T^{*}$. Consider the restriction $T^{*}: L_{1}(\mu) \rightarrow$ $X^{* *}$. As a weakly compact operator, this operator is representable [1, III. 1]. Accordingly there exists bounded measurable function $g: \Omega \rightarrow X^{* *}$ such that $T^{*}(f)=($ Bochner- $) \int_{\Omega} f g d \mu$ for all $f$ in $L_{1}(\mu)$. In particular if $E \in \Sigma$ and $x^{*} \in X^{*}$, then

$$
\begin{aligned}
\int_{E}\left\langle x^{*}, f\right\rangle d \mu & =\int_{\Omega}\left\langle x^{*}, f\right\rangle \chi_{E} d \mu \\
& =\left\langle T^{*}\left(\chi_{E}\right), x^{*}\right\rangle \\
& =\left\langle\int_{E} g d \mu, x^{*}\right\rangle \\
& =\int_{E}\left\langle g, x^{*}\right\rangle d \mu .
\end{aligned}
$$


Since this equality obtains for all $x^{*}$ in $X^{*}$ and $E$ in $\Sigma$, it follows that $f$ is weak*-equivalent to $g$.

To prove the second assertion suppose $f$ is Pettis integrable. Since $g$ is Bochner integrable, we see that

$$
\begin{aligned}
\left\langle x^{*}, P-\int_{E} f d \mu\right\rangle & =\int_{E}\left\langle x^{*}, f\right\rangle d \mu \\
& \left.=\int_{E}\left\langle g, x^{*}\right\rangle d \mu=(\text { Bochner }-) \int_{E} g d \mu, x^{*}\right\rangle
\end{aligned}
$$

for all $E$ in $\Sigma$ and all $x^{*}$ in $X^{*}$. It follows that (Bochner-) $\int_{E} g d \mu \epsilon$ $X$ for every $E$. From this and the argument used to prove [1, III. 3.2] we see that $g$ has almost all of its values in $X$. Redefine $g$ to be zero on the exceptional set and observe that $\left\langle x^{*}, g\right\rangle=\left\langle x^{*}, f\right\rangle$ a.e., for all $x^{*}$ in $X^{*}$. The completed the proof.

One question knocking about for the last couple of years was whether a bounded weakly measurable function $f: \Omega \rightarrow X$ is Pettis integrable if the family $\left\{\left\langle x^{*}, f\right\rangle:\left\|x^{*}\right\| \leqq 1\right\}$ is $L_{\infty}(\mu)$-relatively weakly compact. The truth is that this question had been solved before it was asked. In 1940, Phillips [3] gave an example of a non-Pettis integrable function $f:[0,1] \rightarrow l_{\infty}[0,1]$ that is bounded and weakly measurable and has the property that $\left\{\left\langle x^{*}, f\right\rangle: x^{*} \in\left(l_{\infty}[0,1]\right)^{*}\right\}$ is a one-dimensional subspace of $L_{\infty}(\mu)$. It follows directly that this function is not weakly equivalent to a measurable function, and this shows the assumption of Pettis integrability cannot be dropped in Theorem 2.

The next theorem sets up a link between weak equivalence to a measurable function and universal Pettis integrability. Although the Pettis integral is usually defined with respect to countably additive measures, the definition directly extends to finitely additive measures. Familiar properties of the integral remain unchanged in this new context. For example, if $f: \Omega \rightarrow X$ is Pettis integrable with respect to the finitely additive measure $\lambda$ and if $T: X \rightarrow Y$ is a bounded linear operator, then $T \circ f$ is Pettis integrable and

$$
\int_{E} T \circ f d \mu=T\left(\int_{E} f d \mu\right) \text {. }
$$

THEOREM 3. A bounded weakly measurable function $f: \Omega \rightarrow X$ is weakly equivalent to a measurable function if and only if for each set $B$ in $\Sigma$ with $\mu(B)>0$ there is a subset $A$ of $B$ with $\mu(A)>0$ such that $f \chi_{A}$ is $\lambda$-Pettis integrable for all $\lambda$ in $\left(L_{\infty}(\mu)\right)^{*}$.

Proof. Assume $f$ is weakly equivalent to a measurable function $g: \Omega \rightarrow X$. Fix $B$ in $\Sigma$ with $\mu(B)>0$. Take a sequence $\left(g_{n}\right)$ of 
simple $X$-valued functions that converges to $g \mu$-almost everywhere. With the help of Egorov's Theorem, choose a subset $A$ of $B$ with $\mu(A)>0$ such that the convergence is uniform on $A$. It is now clear that $g \chi_{A}$, as the uniform limit of simple functions, is integrable (in the sense of Dunford and Swartz [2]) with respect to every $\lambda$ in $\left(L_{\infty}(\mu)\right)^{*}$. Now fix $\lambda$ in $\left(L_{\infty}(\mu)\right)^{*}$, fix $E$ in $\Sigma$ and fix $x^{*}$ in $X^{*}$ and note that

$$
\begin{aligned}
\int_{E \cap A}\left\langle x^{*}, f\right\rangle d \lambda & =\int_{E \cap A}\left\langle x^{*}, g\right\rangle d \lambda \\
& =\left\langle x^{*}, \int_{E \cap A} g d \lambda\right\rangle .
\end{aligned}
$$

It follows directly that

$$
\text { Pettis }-\int_{E \cap A} f d \lambda=\int_{E \cap A} g d \lambda
$$

for every $E$ in $\Sigma$. This proves the necessity.

To prove the converse, use Lemma 1 and Theorem 2 to see that for each $B$ in $\Sigma$ with $\mu(B)>0$ there is a subset $A$ of $B$ with $\mu(A)>0$ such that $f \chi_{A}$ is weakly equivalent to a measurable function $g_{A}: \Omega \rightarrow X$. By a standard exhaustion argument [1, III. 2.4] we can find a disjoint sequence $\left(A_{n}\right)$ in $\Sigma$ whose union is $\Omega$ such that for each positive integer $n$ there is a measurable $g_{n}: \Omega \rightarrow X$ such that $f \chi_{A_{n}}$ is weakly equivalent to $g_{n}$. Define $g: \Omega \rightarrow X$ by $g(\omega)=g_{n}(\omega)$ if $\omega \in A_{n}$ and observe that $g$ is measurable and that $f$ is weakly equivalent to $g$. This completes the proof.

2. The family $\left\{x^{*} f:\left\|x^{*}\right\| \leqq 1\right\}$ as a subset of $B(\Sigma)$. Again in this section we shall study the family $\left\{\left\langle x^{*}, f\right\rangle:\left\|x^{*}\right\| \leqq 1\right\}$ for a bounded weakly measurable $f: \Omega \rightarrow X$, but this time we shall study it as a subset of $B(\Sigma)$, the space of all bounded measurable functions on $\Omega$ equipped with the supremum norm. In the last section we saw that $L_{\infty}(\mu)$-relative weak compactness of $\left\{\left\langle x^{*}, f\right\rangle:\left\|x^{*}\right\| \leqq 1\right\}$ is not quite enough to force $f$ to be weakly equivalent to a measurable function. We shall see that the more stringent requirement that $\left\{\left\langle x^{*}, f\right\rangle:\left\|x^{*}\right\| \leqq 1\right\}$ be relatively weakly compact in $B(\Sigma)$ is enough.

THEOREM 4. Let $f: \Omega \rightarrow X$ be bounded and weakly measurable. If $\left\{\left\langle x^{*}, f\right\rangle:\left\|x^{*}\right\| \leqq 1\right\}$ is relatively weakly compact in $B(\Sigma)$, then $f$ is weakly equivalent to a measurable function $g: \Omega \rightarrow X$.

Proof. The relative weak compactness of $\left\{\left\langle x^{*}, f\right\rangle:\left\|x^{*}\right\| \leqq 1\right\}$ in $B(\Sigma)$ patently guarantees the relative weak compactness of this family in $L_{\infty}(\mu)$. A glance at the second part of Theorem 2 shows 
that we will be done once we have shown that $f$ is Pettis integrable. To this end, let $E \in \Sigma$ and $x^{*} \in X^{*}$ and consider the functional

$$
x^{*} \longrightarrow \int_{E}\left\langle x^{*}, f\right\rangle d \mu
$$

defined on $X^{*}$. If we can show this functional is weak*-continuous, then we shall be done, for in this case there is $x_{E}$ in $X$ such that

$$
\left\langle x^{*}, x_{E}\right\rangle=\int_{E}\left\langle x^{*}, f\right\rangle d \mu
$$

for all $x^{*}$ in $X^{*}$. To show this functional is weak*-continuous, it is enough by [2, V. 5.6] to show that this functional is weak*-continuous on the closed unit ball. To this end, let $\left(x_{\alpha}^{*}\right)$ be a net in the closed unit ball of $X^{*}$ that converges weak* to some $x^{*}$ with $\left\|x^{*}\right\| \leqq 1$. The net $\left\langle x_{\alpha}^{*}, f\right\rangle$ then converges pointwise to $\left\langle x^{*}, f\right\rangle$. Since $\left\langle x_{\alpha}^{*}, f\right\rangle$ is a net in a weakly compact subset of $B(\Sigma)$, it follows that $\lim _{\alpha}\left\langle x_{\alpha}^{*}, f\right\rangle=$ $\left\langle x^{*}, f\right\rangle$ weakly in $B(\Sigma)$. Since $\mu \in B(\Sigma)^{*}$, we have

$$
\lim _{\alpha} \int_{E}\left\langle x_{\alpha}^{*}, f\right\rangle d \mu=\int_{E}\left\langle x^{*}, f\right\rangle d \mu .
$$

This proves that the functional

$$
x^{*} \longrightarrow \int_{E}\left\langle x^{*}, f\right\rangle d \mu
$$

is weak*-continuous on bounded subsets of $X^{*}$. This completes the proof.

CoRollary 5. Let $f: \Omega \rightarrow X^{*}$ be a bounded weak*-measurable function. If $\{\langle f, x\rangle:\|x\| \leqq 1\}$ is a relatively weakly compact set in $B(\Sigma)$, then $f$ is weakly measurable and there is a measurable $g: \Omega \rightarrow$ $X^{*}$ such that $f$ is weakly equivalent to $g$.

Proof. Take $x^{* *}$ in the unit ball $X^{* *}$ and pick a net $\left(x_{\alpha}\right)$ in the unit ball of $X$ such that $\lim _{\alpha} x_{\alpha}=x^{* *}$ in the weak*-topology. Then the net $\left(\left\langle f, x_{\alpha}\right\rangle\right)$ converges pointwise to $\left\langle x^{* *}, f\right\rangle$. Since $\left(\left\langle f, x_{\alpha}\right\rangle\right)$ is a net in a weakly compact of $B(\Sigma)$, it follows that $\left\langle x^{* *}, f\right\rangle$ belongs to $B(\Sigma)$ and $\lim _{\alpha}\left\langle f, x_{\alpha}\right\rangle=\left\langle x^{* *}, f\right\rangle$ weakly in $B(\Sigma)$. It follows directly that

$$
\left\{\left\langle x^{* *}, f\right\rangle:\left\|x^{* *}\right\| \leqq 1\right\}
$$

is relatively weakly compact in $B(\Sigma)$.

Local (in the style of the statement of Theorem 3) converses to Theorem 4 and Corollary 5 are also true and are easily proved with 
the help of Egorov's theorem. The global converses fail as the following example demonstrates.

EXAMPLe 6 . Let $\mu$ be Lebesgue measure on the Borel measurable subsets of $(0,1)$ and let $A_{n}=[1 /(n+1), 1 / n)$ for $n=1,2, \cdots$. Let $\left(e_{n}\right)$ be the unit vector basis of $l_{1}$ and define $f:(0,1) \rightarrow l_{1}$ by $f(t)=e_{n}$ for $t$ in $A_{n}$ and note that $f$ is measurable. Letting $\Sigma$ be the Borel subsets of $(0,1)$, one sees that there is an obvious isomorphism from $l_{\infty}$ into $B(\Sigma)$ under which $\left\{\left\langle x^{* *}, f\right\rangle: x^{* *} \in l_{\infty},\left\|x^{* *}\right\| \leqq 1\right\}$ is isomorphic to the unit ball of the unit ball of $l_{\infty}$ and $\left\{\langle x, f\rangle: x \in c_{0},\|x\| \leqq 1\right\}$ is isomorphic to the unit ball of $c_{0}$. Evidently neither of these sets is relatively weakly compact in $B(\Sigma)$.

Replacing the weak compactness condition by a separability condition leads to a characterization of functions that measurable with respect to Radon measures.

THEOREM 7. Let $\Omega$ be a compact Hausdorff space and let $\Sigma$ be the $\sigma$-field of Borel subsets of $\Omega$. Let $\mu$ be a regular Borel measure

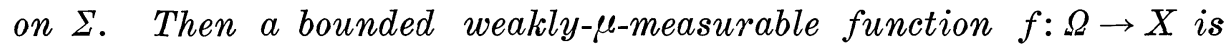
$\mu$-measurable if and only if for each $A$ in $\Sigma$ with $\mu(A)>0$ there is a Borel set $B$ with $B \cong A$ with $\mu(B)>0$ such that the family

$$
\left\{\left\langle x^{*}, f \chi_{B}\right\rangle:\left\|x^{*}\right\| \leqq 1\right\}
$$

is separable in $B(\Sigma)$.

Proof. Suppose $f: \Omega \rightarrow X$ is $\mu$-measurable and $A$ is a Borel set of positive $\mu$-measure. Let $\left(f_{n}\right)$ be a sequence of $X$-valued simple Borel measurable functions that converges to $f \mu$-almost everywhere. By Egorov's theorem there is a Borel set $B \cong A$ such that $\mu(B)>0$ and the convergence is uniform on $B$. Evidently the family $\left\{\left\langle x^{*}, f_{n} \chi_{B}\right\rangle:\left\|x^{*}\right\| \leqq 1, n=1,2,3 \cdots\right\}$ is separable in $B(\Sigma)$ and $\left\{\left\langle x^{*}, f \chi_{B}\right\rangle\right.$ : $\left.\left\|x^{*}\right\| \leqq 1\right\}$ is in its closure. Hence $\left\{\left\langle x^{*}, f \chi_{B}\right\rangle:\left\|x^{*}\right\| \leqq 1\right\}$ is separable.

For the converse, a standard exhaustion argument shows that there is a disjoint sequence of Borel sets $\left(A_{n}\right)$ such that $\mu\left(\Omega \backslash \bigcup_{n=1}^{\infty} A_{n}\right)=$ 0 with the property that $\left\{\left\langle x^{*}, f \chi_{A_{n}}\right\rangle:\left\|x^{*}\right\| \leqq 1\right\}$ is separable in $B(\Sigma)$. If it can be shown that $f \chi_{A_{n}}$ is $\mu$-measurable for each $n$, then it will be shown that $f$ is $\mu$-measurable. Thus there is no loss of generality in assuming that $\left\{\left\langle x^{*}, f\right\rangle:\left\|x^{*}\right\| \leqq 1\right\}$ is separable in $B(\Sigma)$. Assuming this, we see that there is a sequence $\left(\phi_{n}\right)$ of bounded scalar valued functions with the property that for any $x^{*}$ in the unit ball of $X^{*}$ there is a subsequence of $\left(\phi_{n}\right)$ that converges to $\left\langle x^{*}, f\right\rangle$ uniformly on $\Omega$. We can and do assume each $\phi_{n}$ is a simple function. For each $n$ write 


$$
\phi_{n}=\sum_{i=1}^{p_{n}} \alpha_{i, n} \chi_{E_{i, n}}
$$

where for each fixed $n\left\{E_{i, n}\right\}_{i=1}^{p_{1}}$ is a partition of $\Omega$ into Borel sets and the $\alpha_{i, n}$ 's are scalars. Let $\varepsilon>0$. With the help of Lusin's theorem pick compact subsets $F_{i, n}$ of $E_{i, n}$ such that $\left.\phi_{n}\right|_{F_{i, n}}$ is continuous for all $n$ and $i$ and such that $\mu\left(\Omega \backslash \bigcup_{i=1}^{p} F_{i, n}\right)<\varepsilon / 2^{n}$. Observe that $\left.\phi\right|_{\cup_{i=1}^{F_{n}} F_{i, n}}$ is continuous. In addition note that if

$$
F_{\varepsilon}=\bigcap_{n=1}^{\infty}\left(\bigcup_{i=1}^{p_{n}} F_{i, n}\right) \text {, }
$$

then $F_{\varepsilon}$ is compact and $\mu\left(\Omega \backslash F_{\varepsilon}\right)<\varepsilon$. Furthermore note that each function $\left.\phi_{n}\right|_{F_{\varepsilon}}$ is continuous. Since for each $x^{*}$ in the unit ball of $X^{*}$ the function $\left\langle x^{*}, f\right\rangle$ is the uniform limit of a subsequence of $\left(\dot{\phi}_{n}\right)$, it follows that $\left.f\right|_{F_{\varepsilon}}$ is weakly continuous. Since the identity on $X$ is weak-to-norm universally Lusin measurable [5, p. 162 Theorem 3] and $\varepsilon>0$ is arbitrary, this proves that $f$ is (Lusin) $\mu$-measurable and completes the proof.

We have not succeeded in generalizing Theorem 7 to general finite measure spaces.

Example 6 gives an example of a Borel measurable function $f$ such that $\left\{\left\langle x^{*}, f\right\rangle:\left\|x^{*}\right\| \leqq 1\right\}$ is not separable in $B(\Sigma)$. On the other hand an easy corollary of the proof of Theorem 7 gives a sufficient condition for universal Lusin measurability.

CoRollary 8. Let $\Omega$ be a compact Hausdorff space and let $\Sigma$ be the $\sigma$-field of Borel subsets of $\Omega$. If $f: \Omega \rightarrow X$ is a function such that $\left\langle x^{*}, f\right\rangle$ is in $B(\Sigma)$ for all $x^{*}$ in $X^{*}$ and the family $\left\{\left\langle x^{*}, f\right\rangle\right.$ : $\left.\left\|x^{*}\right\| \leqq 1\right\}$ is separable in $B(\Sigma)$, then $f$ is universally Lusin measurable.

3. Pettis integrability and regulated functions. This section is devoted to two isolated theorems. The first deals with Pettis integrability; we believe this result to be the only known theorem that gives a sufficient condition for Pettis integrability and is applicable to some functions that are not weakly equivalent to measurable functions. The second result is a simple theorem dealing with regulated functions on $[0,1]$.

TheOREM 9. Let $\Omega$ be a compact Hausdoff space. If $f: \Omega \rightarrow X$ is a bounded function such that $\left\langle x^{*}, f\right\rangle$ is of the first Baire class for each $x^{*}$ in $X^{*}$, then $f$ is Pettis integrable with respect to every regular Borel measure on $\Omega$. 
Proof. Let $E$ be a Borel subset of $\Omega$ and $\mu$ be a regular Borel measure on $\Omega$. The hypothesis together with Alaoglu's theorem guarantees that the family $\left\{\left\langle x^{*}, f\right\rangle:\left\|x^{*}\right\| \leqq 1\right\}$ is a pointwise compact subset of the first Baire class. According to a fundamental theorem of Rosenthal [4, p. 362], if $\left(x_{\alpha}^{*}\right)$ is a net in the unit ball of $X^{*}$ that converges to $x^{*} \in X^{*}$ in the weak*-topology, then

$$
\lim _{\alpha} \int_{E}\left\langle x_{\alpha}^{*}, f\right\rangle d \mu=\int_{E}\left\langle x^{*}, f\right\rangle d \mu .
$$

Hence the functional

$$
x^{*} \longrightarrow \int_{E}\left\langle x^{*}, f\right\rangle d \mu
$$

on $X^{*}$ is weak*-continuous on bounded sets. An appeal to a standard theorem [2, V. 5.6] shows that this functional is weak*-continuous. Hence there exists $x_{E}$ in $X$ such that

$$
\left\langle x^{*}, x_{E}\right\rangle=\int_{E}\left\langle x^{*}, f\right\rangle d \mu
$$

for all $x^{*}$ in $X^{*}$. Since $E$ was arbitrary and so was $\mu$, the proof is complete.

Often functions are proved to be Pettis integrable by proving they are weakly equivalent to Bochner integrable functions. This is not the case with Theorem 9. In fact the next example shows that Theorem 9 is genuinely a theorem about the Pettis integral.

ExAMPle 10. Define $f:[0,1] \rightarrow L_{\infty}[0,1]$ by $f(t)=\chi_{[0, t]}$. Note that if $\lambda$ is in $\left(L_{\infty}[0,1]\right)^{*}$, then $\langle\lambda, f(t)\rangle=\lambda([0, t])$, a function of bounded variation on $[0,1]$. Hence $\langle\lambda, f\rangle$ is of the first Baire class for all $\lambda$ in $\left(L_{\infty}([0,1])\right)^{*}$. By Theorem 9, the function $f$ is Pettis integrable, with respect to every regular Borel measure. It is easily checked that $f$ is not weakly equivalent to any Bochner integrable function (see [1, II. 2.8]).

According to Theorem 9 if $f:[0,1] \rightarrow X$ is a bounded function such that $\left\langle x^{*}, f\right\rangle$ is absolutely continuous for each $x^{*}$ in $X^{*}$, then $f$ is Pettis integrable with respect to Lebesgue measure. The next theorem shows that if it happens that the family $\left\{\left\langle x^{*}, f\right\rangle:\left\|x^{*}\right\| \leqq 1\right\}$ is weakly compact in the space of absolutely continuous functions, then $f$ has a very strong measurability property.

Recall that a function $f:[0,1] \rightarrow X$ is regulated if it is the uniform limit of functions of the form $\sum_{n=1}^{m} x_{n} \chi_{I_{n}}$ where $x_{n} \in X$ and each $I_{n}$ is a subinterval of $[0,1]$. 
The space $A C[0,1]$ is the space of all absolutely continuous functions on $[0,1]$ equipped with the variation norm.

THEOREM 11. Let $f:[0,1] \rightarrow X$ be a bounded function such that $\left\langle x^{*}, f\right\rangle$ is absolutely continuous for each $x^{*}$ in $X^{*}$. If the family $\left\{\left\langle x^{*}, f\right\rangle:\left\|x^{*}\right\| \leqq 1\right\}$ is relatively weakly compact in $A C[0,1]$, then $f$ is a regulated function.

Proof. By the hypothesis and [2, IV. 13.31] the family $\mathscr{F}=$ $\left\{\left\langle x^{*}, f\right\rangle:\left\|x^{*}\right\| \leqq 1\right\}$ is equi-absolutely continuous on $[0,1]$. Let $\varepsilon>0$ and partition $[0,1]$ into subintervals $\left\{I_{n}\right\}$ such that for each $n$ each of $\mathscr{F}$ has total variation less than $\varepsilon$ on $I_{n}$. Evidently this means that the diameter of $f\left(I_{n}\right)$ is less than or equal to $\varepsilon$ for each $n$. Consequently $f$ is regulated.

\section{REFERENCES}

1. J. Diestel and J. J. Uhl, Jr., Vector Measures, Math. Surveys no. 15, Amer. Math. Soc., Providence, 1977.

2. N. Dunford and J. T. Schwartz, Linear Operators, Part I, Interscience, New York, 1958.

3. R. S. Phillips, Integration in a convex linear topological space, Trans. Amer. Math. Soc., 47 (1940), 114-145.

4. Haskell P. Rosenthal, Point-wise compact subsets of the first Baire class, Amer. J. Math., 99 (1977), 362-378.

5. L. Schwartz, Radon measures on arbitrary topological spaces and cylindrical measures, Oxford, 1973.

Received September 25, 1979 and in revised form July 30, 1980. Portions of this material are included in the first-named author's $\mathrm{Ph}$. D. thesis at the University of Illinois. The Second author was supported in part by the National Science Foundation.

OberLin COLLEge

OBERLIN, OH 44074

AND

UNIVERSITY OF ILLINOIS

URBANA, IL 61801 



\section{PACIFIC JOURNAL OF MATHEMATICS}

\section{EDITORS}

DONALD BABBITT (Managing Editor)

University of Galifornia

Los Angeles, California 90024

Hugo RossI

University of Utah

Salt Lake City, UT 84112

C. C. MOORE AND ANDREW OGG

University of California

Berkeley, CA 94720
J. DugundJI

Department of Mathematics University of Southern California Los Angeles, California 90007

R. FinN and J. Milgram Stanford University Stanford, California 94305

\section{ASSOCIATE EDITORS}

R. ARENS

E. F. BeCKenbaCh

B. H. NEUManN

F. WOLF

K. YosHIDA

\section{SUPPORTING INSTITUTIONS}

UNIVERSITY OF ARIZONA

UNIVERSITY OF BRITISH COLUMBIA

CALIFORNIA INSTITUTE OF TECHNOLOGY

UNIVERSITY OF CALIFORNIA

MONTANA STATE UNIVERSITY

UNIVERSITY OF NEVADA, RENO

NEW MEXICO STATE UNIVERSITY

OREGON STATE UNIVERSITY
UNIVERSITY OF OREGON

UNIVERSITY OF SOUTHERN CALIFONIA

STANFORD UNIVERSITY

UNIVERSITY OF HAWAII

UNIVERSITY OF TOKYO

UNIVERSITY OF UTAH

WASHINGTON STATE UNIVERSITY

UNIVERSITY OF WASHINGTON 


\section{Pacific Journal of Mathematics}

\section{Vol. 95, No. $1 \quad$ September, 1981}

John Allen Beachy and William David Blair, On rings with bounded

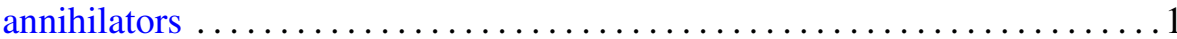

Douglas S. Bridges, A constructive look at positive linear functionals on

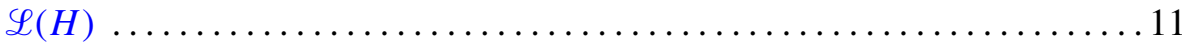

Muneo Chō and Makoto Takaguchi, Boundary points of joint numerical ranges

W. J. Cramer and William O. Ray, Solvability of nonlinear operator equations

Lester Eli Dubins and Gideon Schwarz, Equidiscontinuity of

Borsuk-Ulam functions

Maria Fragoulopoulou, Spaces of representations and enveloping 1.m.c.

*-algebras

Robert F. Geitz and J. Jerry Uhl, Jr., Vector-valued functions as families of scalar-valued functions

Ross Geoghegan, The homomorphism on fundamental group induced by a homotopy idempotent having essential fixed points

Ross Geoghegan, Splitting homotopy idempotents which have essential fixed points

Paul Jacob Koosis, Entire functions of exponential type as multipliers for

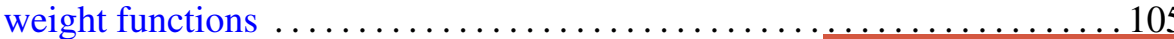

David London, Monotonicity of permanents of certain doubly stochastic matrices

Howard J. Marcum, Two results on cofibers

Giancarlo Mauceri, Zonal multipliers on the Heisenberg group

Edward Wilfred Odell, Jr. and Y. Sternfeld, A fixed point theorem in $c_{0} \quad \ldots 161$

Bernt Karsten Oksendal, Brownian motion and sets of harmonic measure zero

Andrew Douglas Pollington, The Hausdorff dimension of a set of normal numbers

Joe Repka, Base change lifting and Galois invariance ...

Gerald Suchan, Concerning the minimum of permanents on doubly stochastic circulants

Jun-ichi Tanaka, On isometries of Hardy spaces on compact abelian groups

Aaron R. Todd, Quasiregular, pseudocomplete, and Baire spaces 